\title{
ROJ
}

\section{Optimal timing for salvage surgery after definitive radiotherapy in hypopharyngeal cancer}

\author{
Seok-Joo Chun, MD1', Bhumsuk Keam, MD, PhD², Dae Seog Heo, MD, PhD², \\ Kwang Hyun Kim, MD PhD ${ }^{3}$, Myung-Whun Sung, MD, PhD ${ }^{4,5}$, Eun-Jae Chung, MD, PhD \\ Ji-hoon Kim, MD, PhD ${ }^{6}$, Kyeong Cheon Jung, MD, PhD ${ }^{7}$, Jin Ho Kim, MD, PhD ${ }^{1,8,9}$, \\ Hong-Gyun $\mathrm{Wu}, \mathrm{MD}, \mathrm{PhD}^{1,8,9}$
}

Departments of ${ }^{1}$ Radiation Oncology and ${ }^{2}$ Internal Medicine, Seoul National University College of Medicine, Seoul; ${ }^{3}$ Department of Otorhinolaryngology, Bundang Jesaeng Hospital, Seongnam; ${ }^{4}$ Department of Otorhinolaryngology, Seoul National University

College of Medicine, Seoul; ${ }^{5}$ Sensory Organ Research Institute, Medical Research Center, Seoul National University, Seoul; Departments of ${ }^{6}$ Radiology and ${ }^{7}$ Pathology ${ }^{8}$ Cancer Research Institution, Seoul National University College of Medicine, Seoul; ${ }^{9}$ Institute of Radiation Medicine, Medical Research Center, Seoul National University, Seoul, Korea

\begin{abstract}
Purpose: Use of radiotherapy combined with chemotherapy is increasing in hypopharyngeal cancer. However, many show residual tumor after radiotherapy. Timing for treatment evaluation and salvage therapy is essential. However, optimal timing for salvage surgery has not been suggested. In this study, we tried to evaluate optimal timing for salvage surgery.

Methods and Materials: Patients who were diagnosed with hypopharyngeal squamous cell carcinoma between 2006 and 2015 were retrospectively analyzed. All patients received definitive radiotherapy with or without chemotherapy. Response of all treated patients were analyzed at 1, 3, and 6 months after radiotherapy. Any patients with progression before 6 months were excluded.

Results: A total of 54 patients were analyzed. Complete remission (CR) rates at 1 month $\left(\mathrm{CR}_{1}\right), 3$ months $\left(\mathrm{CR}_{3}\right)$ and 6 months $\left(\mathrm{CR}_{6}\right)$ were $66.7 \%, 81.5 \%$, and 90.7\%, respectively. Non-CR at 1 month $(\mathrm{NCR}), 3$ months $\left(\mathrm{NCR}_{3}\right)$, and 6 months $\left(\mathrm{NCR}_{6}\right)$ showed poor locoregional recurrence-free survival rates (1-year rates of $63.7 \%, 66.7 \%$, and $0.0 \%$, respectively) compared to $\mathrm{CR}_{11} \mathrm{CR}_{3}$, and $\mathrm{CR}_{6}(1$ year rates $94.3 \%, 88.0 \%$, and $91.5 \%$, respectively). Particularly significant differences were seen between $C_{6}$ and $N C R_{6}(p<0.001)$. Of 10 patients with $N C R_{3}, 5$ showed $C R$ at 6 months $\left(\mathrm{NCR}_{3} / \mathrm{CR}_{6}\right)$. There was no statistical difference in locoregional recurrence-free survival between $\mathrm{CR}_{3}$ and $\mathrm{NCR}_{3} / \mathrm{CR}_{6}$ group $(\mathrm{p}=0.990)$.

Conclusion: Our data suggest half of patients who did not show CR at 3 months eventually achieved CR at 6 months. Waiting until 6 months after radiotherapy may be appropriate for avoiding additional salvage therapy.
\end{abstract}

Keywords: Hypopharynx, Radiotherapy, Neoplasms

\section{Introduction}

In hypopharyngeal cancer, functional perseveration of the larynx is an important issue in choosing treatment. The European Organization for Research and Treatment of Cancer (EORTC) 24891 study demonstrated that induction

Received 29 June 2018, Revised 24 July 2018, Accepted 06 August 2018.

Correspondence: Hong-Gyun Wu, MD, PhD, Department of Radiation Oncology, Seoul National University College of Medicine, 101 Daehak-ro, Jongno-gu, Seoul 03080, Korea. Tel: +82-2-2072-3177, Fax: +82-2-765-3317, E-mail: wuhg@snu. ac.kr

(c) This is an Open Access article distributed under the terms of the Creative Commons Attribution Non-Commercial License (http://creativecommons.org/ licenses/by-nc/4.0/) which permits unrestricted non-commercial use, distribution, and reproduction in any medium, provided the original work is properly cited.

www.e-roj.org 
chemotherapy with cisplatin and fluorouracil followed by definitive radiotherapy has comparable survival to surgery followed by adjuvant radiotherapy [1]. The Meta-Analysis of Chemotherapy on Head and Neck Cancer (MACH-NC) Collaborative Group conducted a meta-analysis regarding benefits of adding chemotherapy in the treatment of head and neck cancer [2]. For hypopharyngeal cancer, although timing of chemotherapy has failed to show statistical survival benefit concurrent chemoradiotherapy showed significant benefit compared to radiotherapy alone. Based on these data, usage of radiotherapy without surgery is increasing in trend [3-5].

Induction chemotherapy followed by radiotherapy alone or concurrent chemoradiotherapy is now the mainstay of treatment for hypopharyngeal cancer. However, tumor response assessment after radiotherapy is often difficult due to treatment-induced changes such as soft tissue edema, skin thickening and fibrosis. Furthermore, head and neck cancers can regress slowly after treatment, which can be difficult to distinguish from residual tumor [6]. For these reasons, many recommend salvage treatment at least 3 months after definitive radiotherapy. Meanwhile, early detection of residual proliferating tumor cells may allow salvage surgery to be performed without significant complications. Thus, optimal timing for salvage surgery can be crucial in patient management. However, less research is done in hypopharyngeal carcinoma.

In this study, we aim to seek optimal timing for evaluation of patients diagnosed as hypopharyngeal cancers after definitive radiotherapy. Particularly, we aimed to assess response at 1, 3 and 6 months after radiotherapy and show which timepoint may have better association with locoregional failures. By evaluating complete remission (CR) at three timepoints, we could deduce optimal timing of salvage surgery after definitive radiotherapy.

\section{Methods and Materials}

\section{Participants}

Patients who were newly diagnosed as hypopharyngeal cancer from 2006 to 2015 were included. All patients received full dose definitive radiotherapy with or without chemotherapy. Exclusion criteria were as follows: (1) distant metastasis at diagnosis, (2) radical surgery before radiotherapy (however, local excision of hypopharynx and/or neck dissections were allowed), (3) follow-up less than 6 months, (4) previous head and neck radiation history, (5) non-squamous cell histology, and (6) interval locoregional progression within 6 months of follow-up. Patient data were collected retrospectively using electronic medical records and image database. This study was approved by the Health Institutional Review Board of Seoul National University Hospital (No. H-1804-011-932).

\section{Treatment}

Pre-treatment clinical staging was done using laryngoscopy, computed tomography (CT), magnetic resonance imaging (MRI) or positron emission tomography-computed tomography (PET-CT) if possible. After clinical staging, cases were discussed at the multidisciplinary tumor board for head and neck cancers. Aside from the few patients that had early stage hypopharyngeal cancer, most patients were recommended for induction chemotherapy followed by definitive radiotherapy considering larynx function preservation.

Before radiotherapy, CT simulation was done with a thermoplastic immobilization device. All target volumes and organs at risk were contoured on the simulation CT. Gross tumor volume (GTV) consisted of the hypopharyngeal mass and enlarged neck lymph nodes, if any. High-risk clinical target volume $\left(\mathrm{CTV}_{\text {High }}\right.$ ) was defined as GTV plus $5 \mathrm{~mm}$ margins. Intermediate risk CTV (CTV Intermed $_{\text {included areas }}$ of the hypopharyngeal mass expanded with $1 \mathrm{~cm}$ superiorinferior margins and the uninvolved 1st echelon neck lymph nodes. Low-risk CTV $\left(\mathrm{CTV}_{\text {Low }}\right)$ included ipsilateral uninvolved 2nd echelon neck lymph nodes and contralateral neck level II, III lymph nodes in cases with no evidence of contralateral neck metastasis. All CTVs were given $3 \mathrm{~mm}$ margins for planning target volume (PTV). Before 2013, radiotherapy was administrated as three-dimensional conformal radiotherapy (3D-CRT) with shrinking volume technique with dose of $44 \mathrm{~Gy}$ to $\mathrm{PTV}_{\text {Low }} 54 \mathrm{~Gy}$ to $\mathrm{PTV}_{\text {Intermedi }}$ and $70 \mathrm{~Gy}$ to $\mathrm{PTV}_{\text {High }}$ in $2 \mathrm{~Gy}$ dose per fraction. After 2013, simultaneous integrated boost (SIB) technique was commonly used. PTV High, PTV $_{\text {Intermed, }}$ PTV Low was usually prescribed as $67.5 \mathrm{~Gy}, 54 \mathrm{~Gy}$ and $48 \mathrm{~Gy}$ in 30 fractions, respectively.

For chemotherapy, usually induction was done for patients with advanced hypopharyngeal cancer. Regimen contained mainly docetaxel-cisplatin (DP) and few received docetaxel5FU-cisplatin (DFP). At the start of radiotherapy, concurrent chemoradiotherapy was offered in patients with advanced hypopharyngeal cancer who could tolerate treatment, mainly with weekly cisplatin. If patients seemed intolerable to concurrent chemoradiotherapy with cisplatin, cetuximab or no chemotherapy was administered. 


\section{Surveillance and tumor assessment}

Patients were routinely assessed at 2 weeks, 1 month, 3 months, and 6 months after completion of radiotherapy. At 2 weeks, we evaluated acute toxicity induced by radiotherapy without any image findings. For 1-month follow-up, acute toxicity and early response was usually evaluated by laryngoscopy, physical neck examination, and image work up such as CT or MRI. At 3 months and 6 months, clinicians evaluated tumor response with laryngoscopy and all patients went through image work up using $\mathrm{CT}, \mathrm{MRI}$ or PET-CT. The surveillance scheme is described in Fig. 1.

Tumor assessment was categorized into three timepoints (1 month, 3 months, and 6 months). All response assessment at the outpatient clinic was done according to the Response Evaluation Criteria in Solid Tumors (RESIST v1.1) [7]. Response evaluation was classified by a single radiation oncologist with 25 years of experience with head and neck cancer. CR was defined as no suspicious mucosal lesion with normal motility of vocal folds on laryngoscopy and no contrast enhancing or hypermetabolic lesions on image findings. Because partial remission (PR) and stable disease (SD) are difficult to distinguish in hypopharyngeal cancer, we categorized PR and $P D$ as non-CR (NCR). All patients with evident progressive disease (PD) went through salvage operation or chemotherapy depending on patient and disease characteristics. Patients with PD occurring within 6 months of follow-up were offered salvage treatment. These patients were excluded from the final analysis, considering that salvage treatment affects response assessment at 6 months, possibly leading to higher chance of selection bias. For patients with CR at 6 months, no further pathologic confirmation was given per institutional protocol. If suspicious mucosal lesion was sighted, suspension biopsy was done.

\section{Outcomes and statistics}

$\mathrm{CR}_{n}$ was defined as $\mathrm{CR}$ at the $n$th month after radiotherapy. $\mathrm{PR}$ or $\mathrm{SD}$ at the $n$th month were defined as $\mathrm{NCR}_{n}$. For example, $C R$ at 3 months were categorized as $\mathrm{CR}_{3}$, while $P R$ or $S D$ at 6 months were defined as $N_{C R}$. Locoregional failure was defined as signs of PD in head and neck lesions, namely the hypopharynx and regional lymph nodes. Locoregional recurrence-free survival was defined as the base of follow-up to the day when locoregional failure was observed, excluding distant metastasis before locoregional failure. Comparing images between simulation CT and follow-up image, patterns of failure were analyzed according to the radiation field, as either in-field or out-of-field. In-field failures were

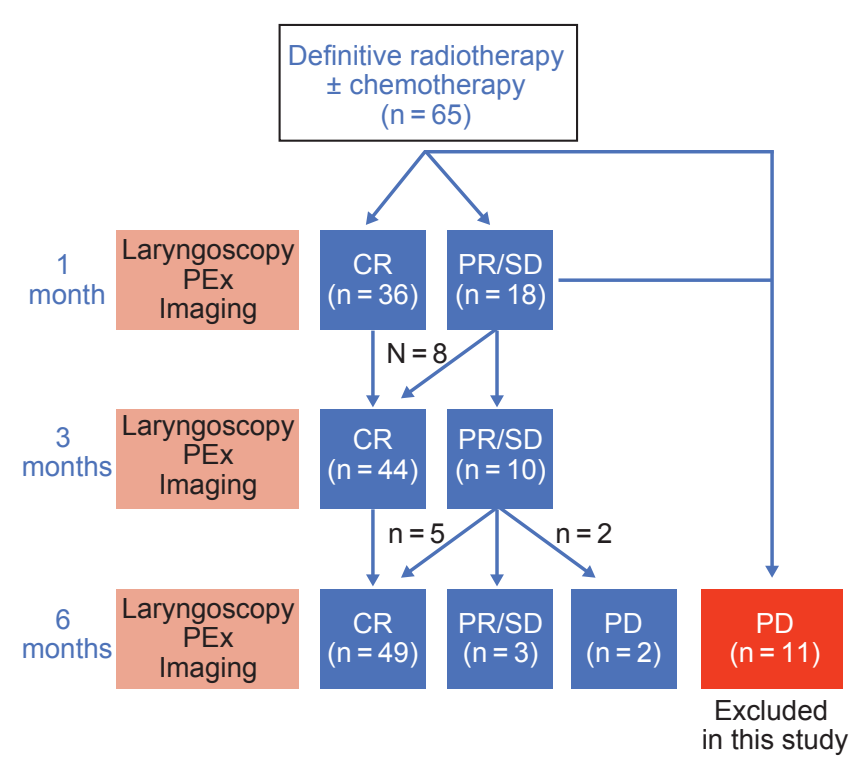

Fig. 1. Surveillance scheme of patients after radiotherapy. $C R$, complete remission; $P R$, partial remission; $S D$, table disease; $P D$, progressive disease.

distinguished by PTV Hight $P T V_{\text {Intermed }}$ and PTV Low areas.

The primary endpoint for this study was locoregional recurrence-free survival. Chi-square analysis for categorical data and Student t-test for continuous variables were used to evaluate any differences between two groups. The KaplanMeier method and the Mantel-Cox log-rank test were used to compare survival data. Cox proportional hazards regression model was used for multivariate analysis of factors, where $p$-value was $<0.1$ in the univariate analysis. Factors with $p<$ 0.05 in each analysis were defined as statistically significant. All the analyses were performed using R 3.4 .3 (http://www. r-project.org/).

\section{Result}

\section{Patient characteristics}

A total of 54 patients were analyzed. The median age was 66.3 years (range, 52.2 to 81.1 years) and majority were males (90.7\%). ECOG performance status was generally good, except 1 patient that had ECOG-PS 2. Induction chemotherapy was delivered in 30 patients (55.6\%) and all received cisplatin-based chemotherapy. DP was used in 15 patients and DFP was used in 7 patients. Nine patients (16.7\%) underwent modified radical neck dissection without total hypopharyngectomy before radiotherapy. Forty-two patients (77.8\%) received concurrent chemoradiotherapy. 
For concurrent chemoradiotherapy, 38 patients received cisplatin as concurrent agents, while 3 received cetuximab and 1 received both cisplatin and cetuximab. The median radiation dose was $67.5 \mathrm{~Gy}$ (range, 63.0 to $70.0 \mathrm{~Gy}$ ). Thirty patients received intensity-modulated radiotherapy (IMRT) and 24 patients received 3D-CRT. Patient characteristics are summarized in Table 1.

\section{Clinical outcomes}

The median follow-up period was 42.3 months (range, 9.4 to 144.4 months). There were 20 locoregional failures during follow-up. Estimated crude locoregional recurrencefree survival at 1 year and 3 years were $84.5 \%$ and $64.1 \%$, respectively. In univariate analysis, age, surgery and RT technique had $p$-value less than 0.1. However, multivariate analysis revealed no statistical significant prognostic factors, with only marginal benefit for surgery $(p=0.081)$. Coxregression analysis of prognostic factors are summarized in Table 2.

\section{Tumor response assessment}

Thirty-six patients showed $C R_{1}$ while 18 showed $N C R_{1}$. Locoregional recurrence-free survival of 1-year and 3-year were $94.3 \%$ and $73.4 \%$ in the $\mathrm{CR}_{1}$ group and $63.7 \%$ and $44.6 \%$ in the $N C R_{1}$ group, respectively $(p=0.036)$. At 3 months, 44 showed $\mathrm{CR}_{3}$, including 8 patients who were $\mathrm{NCR}_{1}$. Locoregional recurrence-free survival at 1 year and 3 years were $88.0 \%$ and $68.3 \%$ in the $\mathrm{CR}_{3}$ group and $66.7 \%$, and $44.4 \%$ in the $\mathrm{NCR}_{3}$ group, respectively $(p=0.080)$. Additional 5 patients who were $\mathrm{NCR}_{3}$ achieved $\mathrm{CR}$ at 6 months. $\mathrm{CR}_{6}$ had favorable outcomes,
Table 1. Clinical characteristics of patients $(n=54)$

\begin{tabular}{lc}
\hline \multicolumn{1}{c}{ Characteristic } & Value \\
\hline Age (yr) & $66.3(52.2-81.1)$ \\
Sex & \\
Male & $49(90.7)$ \\
Female & $5(9.3)$ \\
ECOG performance status & \\
0 & $9(16.7)$ \\
1 & $44(81.5)$ \\
2 & $1(1.8)$ \\
Tstage & \\
T1/T2 & $32(59.3)$ \\
T3/T4 & $22(40.7)$ \\
N stage & \\
No/N1 & $19(35.2)$ \\
N2/N3 & $35(64.8)$ \\
Induction chemoradiotherapy & \\
Yes & $30(55.6)$ \\
No & $24(44.4)$ \\
Surgery & \\
MRND & $9(16.7)$ \\
No & $45(83.3)$ \\
Concurrent chemotherapy & \\
Yes & $42(77.8)$ \\
No & $12(22.2)$ \\
Radiation technique & \\
3D-CRT & \\
IMRT & \\
Radiation dose (Gy) & $30(44.4)$ \\
Varl & $67.5(63.6)$ \\
\hline
\end{tabular}

Values are presented as median (range) or number (\%). ECOG, Eastern Cooperative Oncology Group; MRND, modified radical neck dissection; 3D-CRT, three-dimensional conformal radiotherapy; IMRT, intensity-modulated radiotherapy.

Table 2. Cox-regression analysis of locoregional recurrence-free survival of prognostic factors

\begin{tabular}{|c|c|c|c|c|}
\hline & \multicolumn{2}{|c|}{ Univariate analysis } & \multicolumn{2}{|c|}{ Multivariate analysis } \\
\hline & $\mathrm{HR}(95 \% \mathrm{Cl})$ & p-value & $\mathrm{HR}(95 \% \mathrm{Cl})$ & p-value \\
\hline Age (incremental) & $1.08(1.02-1.14)$ & $0.006^{*}$ & $1.02(0.95-1.09)$ & 0.615 \\
\hline Sex (male vs. female) & $0.69(0.58-6.85)$ & 0.264 & & \\
\hline ECOG performance status (0 vs. 1/2) & $0.73(0.24-2.22)$ & 0.575 & & \\
\hline T stage (T1/2 vs. T3/4) & $0.82(0.35-1.94)$ & 0.655 & & \\
\hline N stage (N0/1 vs. N2/3) & $1.27(0.54-2.98)$ & 0.582 & & \\
\hline Surgery (MRND vs. no) & $3.42(0.80-14.7)$ & $0.076^{*}$ & $6.03(0.80-45.4)$ & $0.081^{*}$ \\
\hline Induction chemoradiotherapy (yes vs. no) & $1.33(0.59-3.02)$ & 0.496 & & \\
\hline Concurrent chemotherapy (yes vs. no) & $1.37(0.57-3.32)$ & 0.479 & & \\
\hline Radiation dose (incremental) & $1.00(1.00-1.00)$ & 0.448 & & \\
\hline Radiation technique (3D-CRT vs. IMRT) & $2.12(0.92-4.95)$ & $0.076^{*}$ & $1.8(0.72-4.56)$ & 0.210 \\
\hline
\end{tabular}

$\mathrm{HR}$, hazard ratio; $\mathrm{Cl}$, confidence interval; ECOG, Eastern Cooperative Oncology Group; MRND, modified radical neck dissection; 3D-CRT, three-dimensional conformal radiotherapy; IMRT, intensity-modulated radiotherapy.

${ }^{*} p<0.1$. 
while all $\mathrm{NCR}_{6}$ patients had locoregional failure within 1 year ( $\mathrm{p}$ $<0.001$ ) (Fig. 2, Table 3).

There were 13 patients who were $\mathrm{NCR}_{1}$ and $\mathrm{CR}_{6}\left(\mathrm{NCR}_{1} / \mathrm{CR}_{6}\right)$. We compared this group with patients with $\mathrm{CR}_{1}$. Difference in locoregional recurrence-free survival between $\mathrm{NCR}_{1} / \mathrm{CR}_{6}$ and $C R_{1}$ were not shown in survival analysis $(p=0.440)$. Also 5 patients who showed $\mathrm{NCR}_{3}$ and $\mathrm{CR}_{6}\left(\mathrm{NCR}_{3} / \mathrm{CR}_{6}\right)$ were compared to $\mathrm{CR}_{3}$ group. There were no statistically significant difference between the two groups ( $p=0.990$ ) (Fig. 3).

Five patient with $\mathrm{NCR}_{6}$ had extremely poor prognosis. Although there was no significant difference in prognostic factors between $\mathrm{CR}_{6}$ and $\mathrm{NCR}_{6}, 3$ patients had T4 tumors and all showed $\mathrm{N} 2 \mathrm{~b}$ or more nodal disease. All had locoregional recurrence with or without distant metastasis within 1 year. Two patients had both locoregional and pulmonary metastasis. Two patients had salvage operation, while 3 received salvage chemotherapy. However, shortly after salvage surgery, 2 patients had progression within the tumor bed and developed lung metastasis. Three patients that received chemotherapy also progressed shortly after. All patients died within 2 years after initial treatment.

\section{Patterns of locoregional failure}

Of the 20 locoregional failures, 14 were isolated local failures, 4 were isolated regional nodal failures, and 2 were both. of the 6 regional nodal failures, failures involved ipsilateral neck level II in 3 patients, ipsilateral neck level III in 1 patient, paraesophageal lymph node in 1 patient, and bilateral neck level II in 1 patient. Among the locoregional failures, 17 were observed in the $\mathrm{PTV}_{\text {High }}$ region (75.0\%), while 2 were in the PTV $V_{\text {Intermed }}$ region and 1 involved both PTV $_{\text {High }}$ and PTV Intermed $_{\text {I }}$ regions. One patient had out-of-field failure (paraesophageal lymph node), but was also accompanied by local failure within the PTV High region.

\section{Discussion and Conclusion}

In this study, 54 hypopharyngeal cancer patients treated by definitive radiotherapy were analyzed. Our data revealed that half of the patients who did not show CR at 3 months resulted to have $C R$ at 6 months. This result implies that closely monitoring residual tumor after radiotherapy up to 6 months might be a reasonable follow-up approach. Meanwhile, residual tumor at 6 months were related with very bad prognosis.

Veterans Affairs (VA) trial and EORTC 24891 trial have shown comparable treatment outcome with 'functional preservation'
A

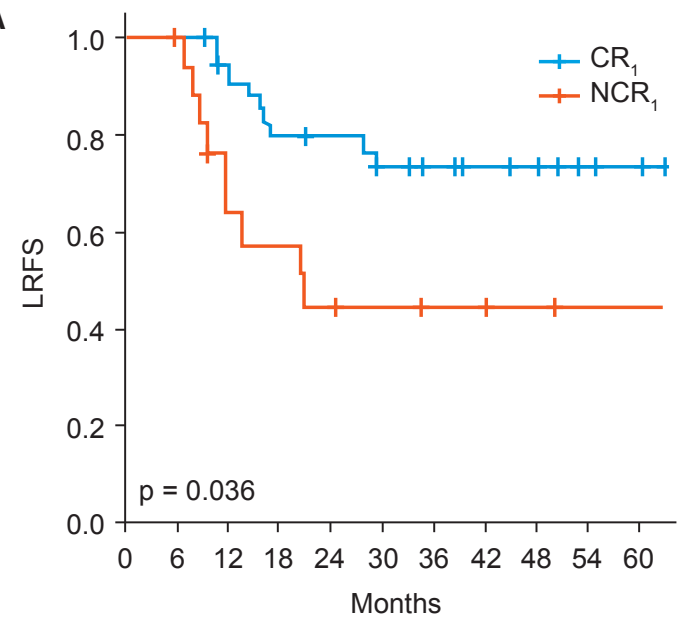

B

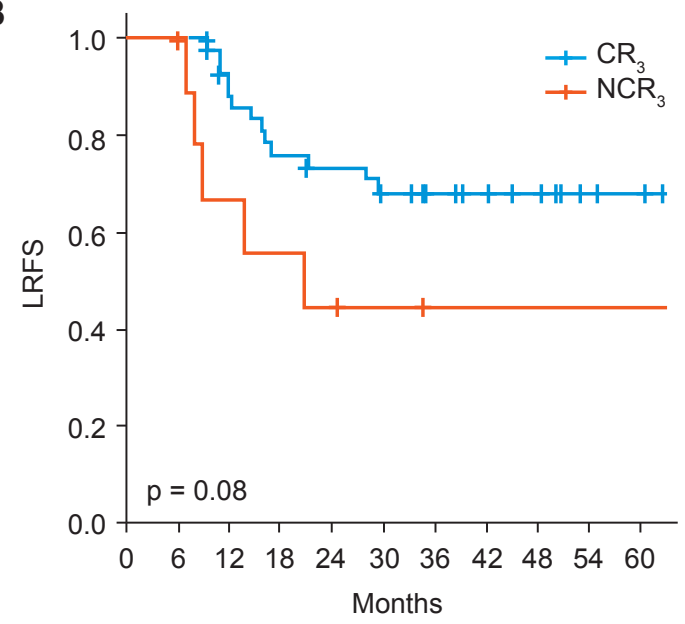

C

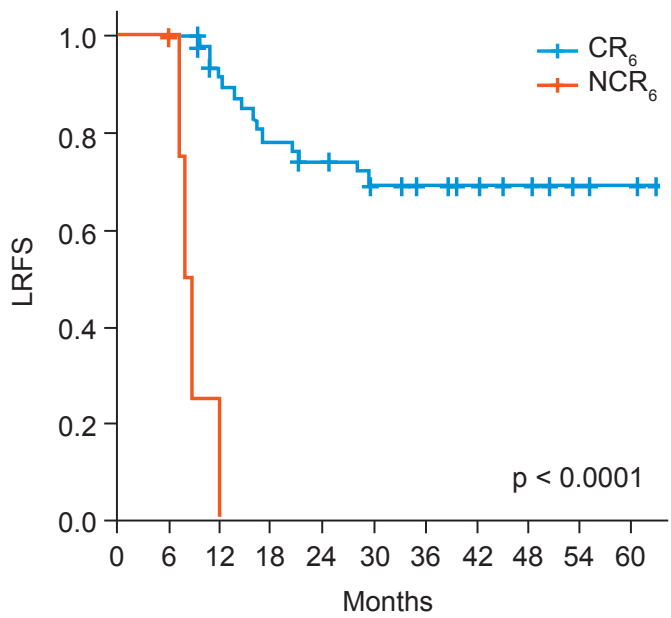

Fig. 2. Kaplan-Meier curve of locoregional recurrence-free rate (LRFS) between complete remission (CR) and non-CR (NCR) at 1 month (A), 3 months (B), and 6 months (C). 
Table 3. Clinical outcomes for tumor response assessment at 1 month, 3 months, and 6 months

\begin{tabular}{|c|c|c|c|c|c|c|}
\hline \multirow{2}{*}{ Assessment } & \multirow{2}{*}{ No. of patients } & \multicolumn{5}{|c|}{ Locoregional recurrence-free survival } \\
\hline & & $1-y r(\%)$ & $3-y r(\%)$ & Crude HR & Adjusted $\mathrm{HR}^{\mathrm{a})}$ & p-value \\
\hline At 1 months & & & & 2.51 & 2.63 & 0.042 \\
\hline $\mathrm{CR}_{1}$ & 36 & 94.3 & 73.4 & & & \\
\hline $\mathrm{NCR}_{1}$ & 18 & 63.7 & 44.6 & & & \\
\hline At 3 months & & & & 2.42 & 1.94 & 0.091 \\
\hline $\mathrm{CR}_{3}$ & 44 & 88.0 & 68.3 & & & \\
\hline $\mathrm{NCR}_{3}$ & 10 & 66.7 & 44.4 & & & \\
\hline At 6 months & & & & 34.2 & 24.2 & $<0.001$ \\
\hline $\mathrm{CR}_{6}$ & 49 & 91.5 & 69.4 & & & \\
\hline $\mathrm{NCR}_{6}$ & 5 & 0.0 & 0.0 & & & \\
\hline
\end{tabular}

$\mathrm{HR}$, hazard ratio; $\mathrm{CR}_{n}$ complete remission in $n$-th month; $\mathrm{NCR}_{n}$, non-complete remission in $n$-th month.

${ }^{a}$ Adjusted for confounding factors (age, radiotherapy technique, surgery).

using induction chemotherapy followed by radiotherapy $[1,8]$. Following results of these landmark trials, usage of radiotherapy combined with chemotherapy has increased for hypopharyngeal and laryngeal cancer [3-5]. However, due to the biologic nature of radiotherapy, there has been difficulties in assessing tumor response. Historically, otolaryngologic examination combined with physical examinations were commonly used for evaluation. More recently, image modalities such as MRI and PET-CT showed good sensitivity and specificity especially in assessing nodal response $[9,10]$. Based on findings mainly from PET-CT, individualized salvage treatment was provided.

A

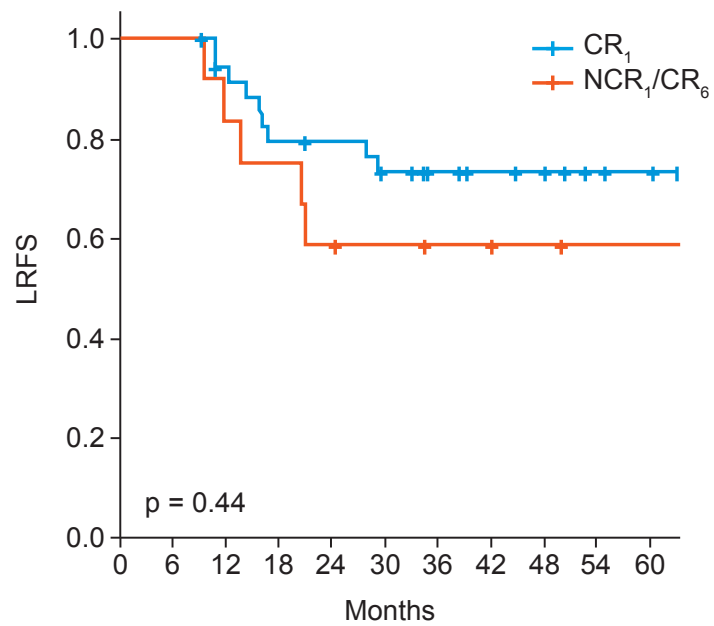

With advance of image findings, question may arise concerning when it would be appropriate to assess tumor response. Although there is no direct randomized trial solely done for hypopharyngeal cancer regarding early surgery versus active surveillance, many trials were conducted comparing these options in head and neck cancers in general. Mehanna et al. [11] conducted a randomized prospective trial comparing PET-CT and early neck dissection at 12 weeks after completion of chemoradiotherapy. In this trial, overall survival was comparable between the PET-CT group and early neck dissection group. Moreover, approximately $80 \%$ of patients in the PET-CT group were spared of neck dissection, showing CR.

\section{B}

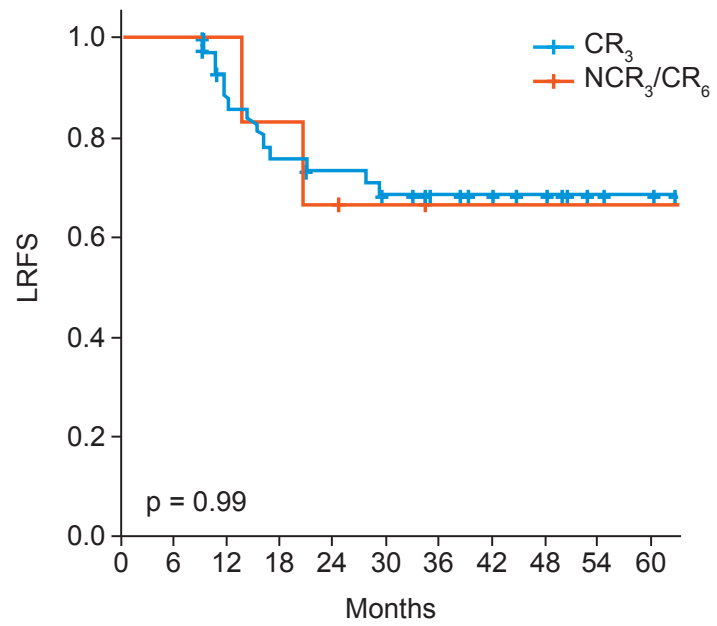

Fig. 3. Kaplan-Meier curve of locoregional recurrence-free rate (LRFS) between (A) CR at 1 month $\left(C R_{1}\right)$ and non-CR at 1 month with $C R$ at 6 months $\left(\mathrm{NCR}_{1} / \mathrm{CR}_{6}\right)$ and $(\mathrm{B}) \mathrm{CR}$ at 3 months $\left(\mathrm{CR}_{3}\right)$ and non-CR at 3 months with $\mathrm{CR}$ at 6 months $\left(\mathrm{NCR}_{3} / \mathrm{CR}_{6}\right)$. CR, complete remission. 
Traditionally, timing of image modality was done around 1216 weeks after radiotherapy, including aforementioned trials $[9,11,12]$. However, Huang et al. [6] reported nodal CR was observed even 6 months after radiotherapy. This is consistent with our results. Two-thirds who were $\mathrm{NCR}_{1}$ changed into $\mathrm{CR}_{6}$. Also, one half of patients who had $\mathrm{NCR}_{3}$ turned to $\mathrm{CR}_{6}$. This implies even residual disease at early tumor assessment time point have high possibility of regression after short term of follow-up after treatment.

Salvage surgery, which is mainly total hypopharyngectomy with muscle flap, is closely related with poor quality of life $[13,14]$. Also 5 -year overall survival after salvage surgery for recurrent hypopharyngeal cancer has been reported as 20\%-40\% [15-17]. Considering high morbidity and poor prognosis after salvage surgery, optimal timepoint for tumor assessment should be made. Many surgeons advocate early biopsy for residual tumor in short follow-ups, considering early salvage operation when feasible. However, early biopsy which show residual tumor might have high possibility of being necrotic tumor cells after radiotherapy. In our data, 10 patients did not show CR at 3 months. However, 5 had turned to $\mathrm{CR}$ at 6 month. Among them, 4 patients locoregionally had no evidence of disease during follow-up. Also, $\mathrm{CR}_{3}$ and $\mathrm{NCR}_{3} /$ $\mathrm{CR}_{6}$ showed comparable locoregional recurrence-free survival. Thus, early salvage surgery for these patients would have rather been harmful.

Meanwhile, 5 patients who were $\mathrm{NCR}_{6}$ had extremely poor prognosis. Although our data do not make conclusion for future treatment for patients who show CR at 6 months, results imply that residual tumor show progression soon after 6 months. Thus, salvage treatment should be considered for patients who show residual disease after 6 months. Since it is reasonable that salvage therapy should be done for patients with evident signs of progression within 6 months, we recommend timing of salvage therapy according to Fig. 4.

The limitation of this study lies in its retrospective nature with small numbers in a single institution. As stated above, complexities in tumor response assessment after definitive radiotherapy could possibly show intra-observer and interobserver variability. Also at post-radiation status, PET-CT, which has an important role in primary and nodal tumor response assessment, were not routinely used in the 2000s. There may have been possible bias in assessing tumor response without PET-CT. HPV or p16, known as highly radiosensitive factors, was not routinely done in the late 2000s in our institution. As only one-third in our cohort had known HPV status, we could not analyze the difference between HPV-positive and HPV- negative cancer.

In conclusion, our data suggest that many who did not show CR at 1 or 3 months show CR at 6 months. Earlier assessment with early salvage treatment may bring unnecessary surgery. Our data suggests waiting for tumor regression until 6 months after radiotherapy may be appropriate.

\section{Conflict of Interest}

No potential conflict of interest relevant to this article was reported.

\section{Acknowledgments}

This study was supported by the National RetD Program for Cancer Control, Ministry of Health and Welfare, Republic of

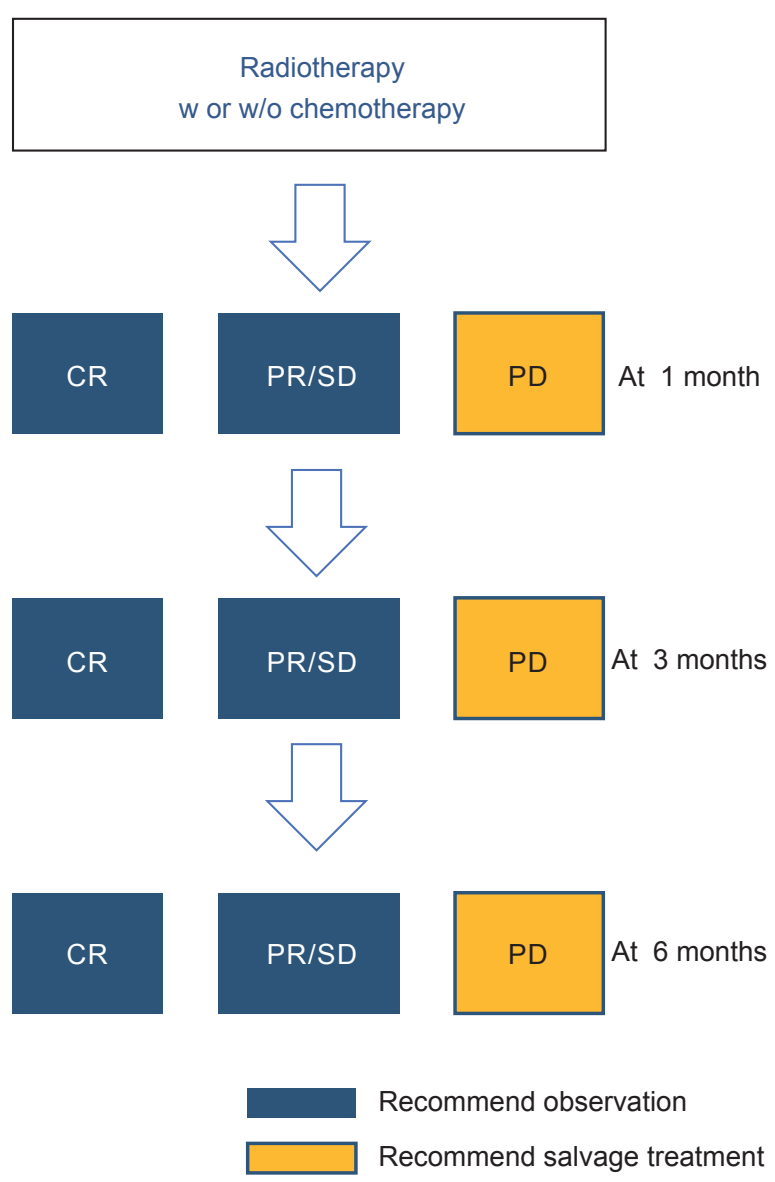

Fig. 4. Recommendations for timing of salvage treatment. $C R_{\text {, }}$ complete remission; $P R$, partial remission; $S D$, table disease; $P D_{\text {, }}$ progressive disease. 
Korea (No. 1631200), and National R\&D Program through the Dong-Nam Institute of Radiological \&t Medical Sciences funded by the Ministry of Education, Science and Technology (No. 50595-2018).

\section{References}

1. Lefebvre JL, Andry G, Chevalier D, et al. Laryngeal preservation with induction chemotherapy for hypopharyngeal squamous cell carcinoma: 10-year results of EORTC trial 24891. Ann Oncol 2012;23:2708-14.

2. Pignon JP, le Maitre A, Maillard E, Bourhis J; MACH-NC Collaborative Group. Meta-analysis of chemotherapy in head and neck cancer (MACH-NC): an update on 93 randomised trials and 17,346 patients. Radiother Oncol 2009;92:4-14.

3. Newman JR, Connolly TM, Illing EA, Kilgore ML, Locher $J L$, Carroll WR. Survival trends in hypopharyngeal cancer: a population-based review. Laryngoscope 2015;125:624-9.

4. Silver CE, Beitler JJ, Shaha AR, Rinaldo A, Ferlito A. Current trends in initial management of laryngeal cancer: the declining use of open surgery. Eur Arch Otorhinolaryngol 2009;266:1333-52.

5. Takes RP, Strojan $P$, Silver $C E$, et al. Current trends in initial management of hypopharyngeal cancer: the declining use of open surgery. Head Neck 2012;34:270-81.

6. Huang SH, O'Sullivan $B, X u W$, et al. Temporal nodal regression and regional control after primary radiation therapy for N2N3 head-and-neck cancer stratified by HPV status. Int J Radiat Oncol Biol Phys 2013;87:1078-85.

7. Eisenhauer EA, Therasse P, Bogaerts J, et al. New response evaluation criteria in solid tumours: revised RECIST guideline (version 1.1). Eur J Cancer 2009;45:228-47.

8. Department of Veterans Affairs Laryngeal Cancer Study Group. Induction chemotherapy plus radiation compared with surgery plus radiation in patients with advanced laryngeal cancer. N Engl J Med 1991;324:1685-90.
9. Wang YF, Liu RS, Chu PY, et al. Positron emission tomography in surveillance of head and neck squamous cell carcinoma after definitive chemoradiotherapy. Head Neck 2009;31:44251.

10. Zundel MT, Michel MA, Schultz CJ, et al. Comparison of physical examination and fluorodeoxyglucose positron emission tomography/computed tomography 4-6 months after radiotherapy to assess residual head-and-neck cancer. Int J Radiat Oncol Biol Phys 2011;81:e825-32.

11. Mehanna H, Wong WL, McConkey CC, et al. PET-CT surveillance versus neck dissection in advanced head and neck cancer. N Engl J Med 2016;374:1444-54.

12. Kapoor V, Fukui MB, McCook BM. Role of 18 FFDG PET/CT in the treatment of head and neck cancers: principles, technique, normal distribution, and initial staging. AJR Am J Roentgenol 2005;184:579-87.

13. Boscolo-Rizzo P, Maronato F, Marchiori C, Gava A, Da Mosto MC. Long-term quality of life after total laryngectomy and postoperative radiotherapy versus concurrent chemoradiotherapy for laryngeal preservation. Laryngoscope 2008;118:300-6.

14. Hanna E, Sherman A, Cash D, et al. Quality of life for patients following total laryngectomy vs chemoradiation for laryngeal preservation. Arch Otolaryngol Head Neck Surg 2004;130:8759.

15. Stoeckli SJ, Pawlik AB, Lipp M, Huber A, Schmid S. Salvage surgery after failure of nonsurgical therapy for carcinoma of the larynx and hypopharynx. Arch Otolaryngol Head Neck Surg 2000;126:1473-7.

16. Taki $S$, Homma A, Oridate $N$, et al. Salvage surgery for local recurrence after chemoradiotherapy or radiotherapy in hypopharyngeal cancer patients. Eur Arch Otorhinolaryngol 2010;267:1765-9.

17. Wong LY, Wei WI, Lam LK, Yuen AP. Salvage of recurrent head and neck squamous cell carcinoma after primary curative surgery. Head Neck 2003;25:953-9. 